\section{Search Using Multiple UAVs with Flight Time Constraints}

\author{
P. B. SUJIT, Student, IEEE \\ D. GHOSE, Member, IEEE \\ Indian Institute of Science
}

\begin{abstract}
We consider a large scale system consisting of multiple unmanned aerial vehicles (UAVs) performing a search and surveillance task, based on the uncertainty map of an unknown region. The search algorithm is based on the $k$-shortest path algorithm that maximizes the effectiveness of the search in terms of searching through the maximum uncertainty region, given a constraint on the endurance time of the UAV and on the location of the base station from which the UAVs operate. These constraints set apart this class of problems from the usual search and surveillance problems. We compare the performance of this algorithm with a random search and a greedy strategy search. We also implement the algorithm for the case of multiple UAVs searching an unknown region. The cases of delayed and partial information are also considered. Simulation results that demonstrate the efficacy of the technique are also presented.
\end{abstract}

Manuscript received January 23, 2003; revised September 11, 2003; released for publication December 13, 2003.

IEEE Log No. T-AES/40/2/831361.

Refereeing of this contribution was handled by K. C. Chang.

This work was supported by the IISc-DRDO program on advanced research in mathematical engineering.

Authors' address: Dept. of Aerospace Engineering, Indian Institute of Science, Bangalore 560 012, India, E-mail: (dghose@aero.iisc.ernet.in).

0018-9251/04/\$17.00 (c) 2004 IEEE

\section{INTRODUCTION}

Searching for targets in an unknown environment has been an area of intense research activity in the search theory literature for several decades [1]. However, much of the work in this area has been somewhat theoretical in nature. Recent efforts to improve the efficacy of search and surveillance operations using a large scale system of multiple unmanned (or uninhabited) aerial vehicles (UAVs) has again brought this area of work to the forefront. Typically, such operations have applications in military operations where interaction between components of large and complex systems are very common [2]. One specific application that has recently emerged due to advances in computing, wireless communications, and vehicular technologies, is the task of large scale deployment of multiple UAVs that can gather information about a dynamic threat/target environment, evade threats, and coordinate strikes against targets [3-5]. In this context we address the problem of a team of UAVs searching for targets in a search space. Passino et al.'s [6] is a seminal paper in this direction in the sense that it lays down a framework in which the behavior of a team or swarm of UAVs can be studied under various operational conditions, strategies, and task goals.

The problem of searching an unknown environment has been addressed in classical search theory [7], except for the fact that there are several realistic considerations (such as communication constraint, autonomy in decision making capability, information constraint, multiplicity of searchers, and distributed decision making issues) that are normally not taken into account in the classical search theory literature. In this regard, developments in this area will not only be significant in terms of ready and pragmatic applications, but will also contribute to the advances in search theory.

Search theory is one of the oldest areas of operations research. The classical work on search theory has mainly concentrated on the problems of optimal distribution of search effort, maximization of the probability of detection of a stationary target, and establishment of optimal values of various search parameters [7-9]. Modern research on search theory has expanded the domain of search theory by considering moving targets, multiple targets, intelligent targets (where the search problem is formulated in a game-theoretical framework), and so on [1]. However, all the above studies primarily dealt with the problem of search by a single searcher, and hence the models are not suitable for applications where a team of agents are searching for targets or trying to accomplish a task. Some notable exceptions, recently published, are the following. In [3] the problem of searching a wide area in a systematic fashion until the detection of a target, using multiple 
vehicles, is addressed. The total path length covered by the searching vehicles is minimized while exhaustively searching the area. The approach depends on prior definition of lanes for each vehicle and takes into account practical aspects like wind disturbances and existence of decoys and the resulting delays in the flight plans. However, uneven probability of target distribution, dynamics in the information about target location, and the role of communication between vehicles are not addressed. In [4], the authors address the important problem of coordinating a rendezvous operation between UAVs. The problem is posed as an optimization problem that maximizes the survival of the UAV team. In [5], the problem of allocating search resources, in terms of swarms of search munitions, over a search area is proposed using a linear programming formulation.

In [10] several mobile robots are assumed to search for targets in a search region. The paper basically addresses the problem of communication between two groups of robots for effective coordination between them. In [11], space-filling curves are used to define open-loop search trajectories for a team of robots carrying out an exhaustive geographical search operation. In [12], a similar problem of exploring an unknown environment using multiple robots is posed and solved using the concept of target points being assigned to each individual robots to maximize search effectiveness. Kitamura et al. [13] deal with multiple agents searching a graph in which link traversal incurs a cost. The cost of the links also change dynamically and the agents need to communicate this information to the other agents to recompute their paths. In [14] multiple searchers searching for a target whose location is known a priori in the form of a probability distribution is addressed as a static path planning problem in discrete state space. Since the problem is of very high complexity (NP hard, to be precise), several heuristic algorithms are proposed.

The basic model for search space that we use is based on a discretization of the search space and search time, somewhat similar to that proposed in [6], where the search region is represented as a collection of cells through which the UAVs pass through. It is assumed that the search time (which also represents the search effort) of the UAVs is partitioned into time periods of equal duration which an UAV spends in a cell that it visits in its route. This does not mean that an UAV distributes its search effort uniformly over the cells that it visits, since the UAV may visit a cell several times in its route and/or may even spend an integer multiple of this time period in a cell. The dynamics of the aerial vehicles are suppressed in these models.

The a priori knowledge of the location of the target is represented as an uncertainty map which

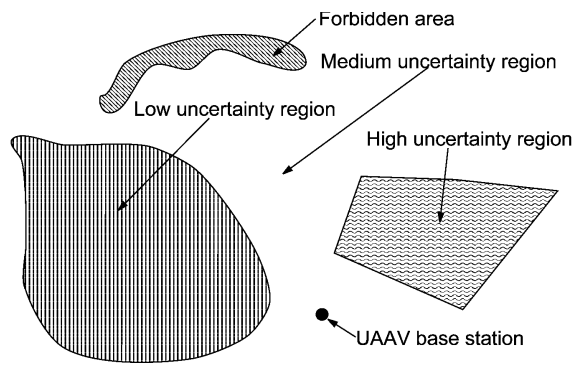

Fig. 1. Hypothetical uncertainty map on search terrain.

could be a function of the probability distribution of the target's location on the cells constituting the search space. An example of a hypothetical uncertainty map, before discretization, on the terrain to be searched is shown in Fig. 1 for illustrative purposes. The uncertainty values associated with each cell reduces as these cells are visited by a UAV. Hence, the objective of an optimal route for a UAV would be to maximize the reduction in uncertainty as it searches the unknown region. The problem, as stated here, is somewhat ill-posed and requires further constraints to make it realistic. We propose a strategy for a UAV to determine optimal search routes for each sortie of the vehicle based on its knowledge of the uncertainty map. We incorporate an endurance time constraint on the UAV, since UAVs are small aerial vehicles that have very limited sojourn time before requiring refueling. This model was first proposed by the authors in [15] where some preliminary results were reported.

\section{PROBLEM FORMULATION}

\section{A. Discretization of Search Area}

We partition a two-dimensional search region into a collection of identical regular hexagonal cells. The reason for using a hexagon as the basic unit is that it offers the flexibility to the searcher to move in six uniformly distributed directions at each time step and reach a neighboring cell while expending the same amount of energy (see Fig. 2). We assume that the cells are large enough to allow the UAVs to maneuver inside them and change their heading to any desirable direction. This will not be possible if the cells are small. One way to interpret this model is to consider the cells that constitute a search route for a UAV as waypoints that are used by the UAV as a reference to determine its flight path and to determine in which region its search effort should be expended. For example, if a route is given as a sequence of cells, then the UAV uses this as a command to visit the region represented by each of these cells in the same sequence, use its sensor to search each cell region, and then continue on to the next cell in the sequence. 


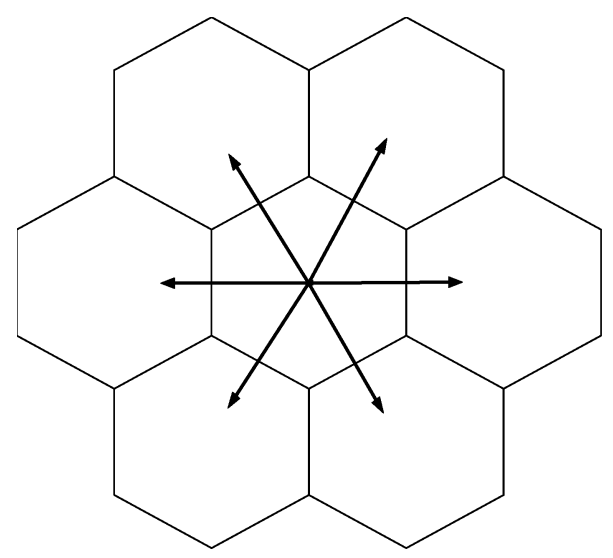

Fig. 2. Partitioning of the search space into hexagonal cells.

\section{B. Uncertainty Map}

The uncertainty map constitutes real numbers between 0 and 1 associated with each cell in the search space. These numbers represent the undetected mass in that cell, or it represents the uncertainty with which the location of the target is known in that cell. A probabilistic interpretation of the uncertainty would be as follows. An uncertainty value of 0.6 would imply that any statement about the target's location in cell $i$ (say) would be true only with probability 0.4 . An uncertainty value of 0 would imply that everything is known about the cell (that is, one can say with certainty whether a target is located in that cell or not). On the other hand, an uncertainty value of 1 would imply that nothing can be said about the location of the target in that cell. Another interpretation of the uncertainty value is that it is the undetected mass in the cell and represents the extent of the lack of information about that cell. Hence, a successful search operation is one that manages to visit those cells that have large uncertainty values.

If a cell is visited for a total of $n_{k-1}$ time steps during the $k-1$ th sortie or mission, then the uncertainty value $U_{k}$ associated with that cell at the beginning of the next sortie ( $k$ th sortie) is given by

$$
U_{k}=(\beta)^{n_{k-1}} U_{k-1}
$$

where $\beta \in[0,1)$ is the uncertainty reduction factor associated with each visit to a cell. This factor $\beta$ is similar to the detection function used in search theory [9], where the detection function represents the probability that a search in a given cell for a specified duration of time will detect the target provided that the target is present in that cell. An exponential detection function, which is normally used in search problems, is represented as $1-e^{\alpha t}$ where $t$ is the time spent in the cell and $\alpha$ is a scaling factor also known as the detection rate. It has the property of diminishing returns in the sense that each incremental time spent in searching a cell produces a decreasing return on the probability of detection.
Note that $\beta$ in (1) has a similar effect in the sense that the incremental reduction in uncertainty with each subsequent visit reduces. Note that $n_{k-1}$ is not necessarily the number of times that a single UAV visits that cell. It could be the total number of times that cell is visited by all the UAVs during the $k-1$ th sortie. It is easy to generalize this to a case when the uncertainty reduction factor is different for each UAV, perhaps due to their differing capability. In which case we have,

$$
U_{k}=\prod_{i=1}^{M}\left(\beta_{i}\right)^{n_{k-1}^{i}} U_{k-1}
$$

where $M$ is the total number of UAVs, $\beta_{i}$ is the uncertainty reduction factor associated with the $i$ th $\mathrm{UAV}$, and $n_{k-1}^{i}$ is the number of times that the $i$ th UAV visited the given cell in the $k-1$ th sortie.

\section{Searcher Objective and Constraints}

Consider a single UAV used to search the unknown region. The UAV carries limited fuel that allows it to fly a limited length of trajectory per sortie and then return to base for refuelling. After refuelling it flies another sortie of the same length. Note that "length" of a sortie is meant to represent only the physical distance that the UAV flies, and has nothing to do with either the "cost" or the "benefit" associated with a route, which is defined later. The objective of the UAV is to maximize the reduction in uncertainty at each sortie. Hence, this reduction in uncertainty forms the benefit associated with a sortie. Since we have split the search space uniformly into identically sized cells, we can represent the energy spent by the UAV in moving from one cell to another as equivalent to one unit step length. We assume that the entire energy of the UAV per sortie is limited to $N$ (say) number of steps. This constraint not only puts a limit on the number of cells that an UAV can search, or equivalently on its flight time, but also a constraint on the path in the sense that it has to take off from, and land back into, the same cell, where essentially the UAV base is located.

Each UAV has to perform several sorties or missions and the number of such search missions is unspecified. Each mission consists of one sortie of the search vehicle, where sortie is a trajectory from source cell to a destination cell. We also consider the possibility that the searcher may devote more search effort in a single cell, because of higher uncertainty value in the cell. This would mean that the UAV may spend more than the duration of one time step to search this cell.

At this point, it is necessary to define what we mean by searching the cell. Each UAV is equipped with some kind of sensor with which it collects data or information about the cell it is visiting. So a UAV that spends a certain number of steps (one or more), 
which is measured in terms of multiples of time units per step, in searching any given cell is essentially spending this time in collecting data about the cell and thus the uncertainty in that cell reduces as a function of the time spent there.

Further, since we assume the cells to be large enough compared with the area needed by the UAV to maneuver, the UAV could either be a rotorcraft or a fixed-wing type. The exact type of the UAV becomes relevant when the cells are small or the design of the actual search trajectories inside a cell is undertaken. Also, since our main objective is to develop a viable algorithm for optimal path generation, we do not model the flight dynamics and sensing capabilities of the UAV as a part of the overall problem formulation. But, certainly this should be addressed in a complete system implementation.

The updating of the uncertainty map is done only after the completion of a sortie or search mission. That is, irrespective of the number of times a cell is visited in any given sortie, the uncertainty associated with the cell is assumed to remain unchanged during the sortie. This condition is valid even if the searcher moves from one cell to another and back to the same cell. The uncertainty map is updated, taking into account the effect of multiple visits, only after the current mission is complete and before the next mission commences. It would be fair to mention that this is a somewhat strong assumption and is not supported by the probabilistic model of the uncertainty map. However, an example of a practical application where this model has a reasonable validity is that of a UAV equipped with sensors that collect and store data on-board. This data is processed after the sortie is complete and the UAV has returned to its base. Based on the results of the data processing, the uncertainty map is updated. Thus, the $\beta_{i}$ in (2) is no longer a constant, but is a function of the results of the data processing operation after the UAV arrives at its base station at the end of a sortie.

If instant communication between UAVs is assumed, it would imply that the UAVs should carry communication equipment, target recognition software, and sufficient intelligence capability. However, in our study we assume the UAVs to carry only a sensor (perhaps optical or otherwise) which is used to obtain information about the objects in a terrain in the form of images or signal maps. These can be processed to gain information about a given cell only after the UAVs has returned to the base. This is a more realistic scenario as the present day UAVs are small and carry at most a camera or some other imaging equipment.

\section{Graph Theoretical Representation of Uncertainty}

The algorithm we propose later is based upon a graph theoretical representation or model of the

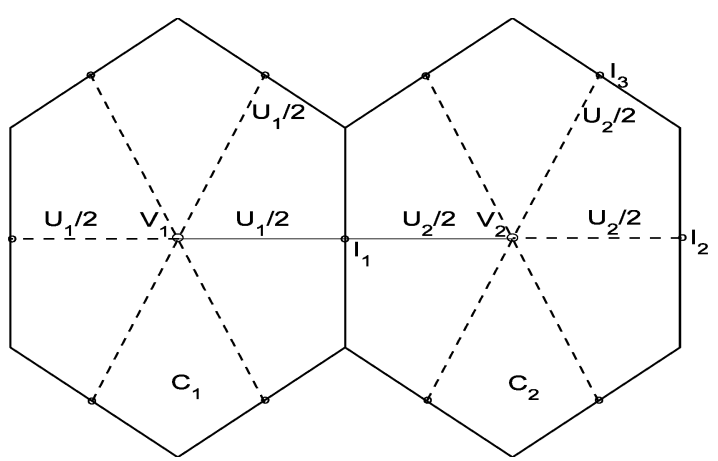

Fig. 3. Graph model of the uncertainty map.

uncertainty map. We consider the center of each hexagonal cell as a cell node or a cell vertex. The path that a UAV takes to traverse from one cell to a neighboring cell has to pass through the boundary between these cells. The uncertainty value is different on one side of the boundary than the other side. Since an edge in a graph cannot have two different values, one can either take average of the two values or create an intermediate node between the two cell nodes. We adopt the latter method since it makes it easier to map on the graph theoretical results to the original search space. Moreover, it is easier to represent looping (that is, a UAV spending more than one time step in the same cell) by paths that go from a cell node to an intermediate node and back again to the cell node (this could be thought of as "chattering"). However, this method also increases the number of vertices in the graph considerably. Hence, for a search area which is partitioned into a collection of a large number of hexagonal cells, it may be advisable to use the former method at the cost of some inaccuracy in computing the uncertainty map. However, we stress that the algorithm proposed here is applicable irrespective of the graph model used.

As an illustration of the graph model adopted here, consider two cells $C_{1}$ and $C_{2}$, having cell nodes $V_{1}$ and $V_{2}$, with associated uncertainty values $U_{1}$ and $U_{2}$ (shown in Fig. 3). The intermediate node between them is termed as $I_{1}$, the edge $\left(V_{1}, I_{1}\right)$ has weight $U_{1} / 2$, and the edge $\left(I_{1}, V_{2}\right)$ has weight $U_{2} / 2$. In the figure, a path segment given by the node sequence $I_{1}, V_{2}, I_{2}$ or $I_{1}, V_{2}, I_{1}$ would constitute one time step equivalent of dwell time in the cell $C_{2}$. A path segment $I_{1}, V_{2}, I_{2}, V_{2}, I_{1}$ or $I_{1}, V_{2}, I_{3}, V_{2}, I_{3}$ would constitute 2 time steps equivalent of dwell time in cell $C_{2}$.

Finally, the graph $G=(V, E)$ constitutes the model for the search space, where $V$ is the set of vertices composed of the cell nodes as well as the intermediate nodes, and $E$ represents the (bidirectional) edges between them. Each cell node has a maximum of six edges leading out of it and each intermediate node has a maximum of two edges. 


\section{OPTIMIZATION PROBLEM AND SOLUTION METHOD}

\section{A. Optimization Problem}

The problem of maximum reduction in uncertainty can be transformed to a constrained optimization problem, wherein we need to find a path $P$ that satisfies the following constraints.

1) Maximize the sum of the reduction in uncertainty values associated with the cells that constitute the path $P$ for each sortie.

2) The path has the constraint that it starts from a source cell and ends at a destination cell, both of which are prespecified. In fact, in this problem we assume the source cell and the destination cell to be the same. In other words, the UAV takes off from its base and after the sortie, returns to the base.

3) Because of the fixed fuel capacity, the UAV has a fixed flight time, which translates to a fixed number of time steps for which the UAV can operate (may also be considered as a fixed number of cells an UAV can visit with multiple visits taken into account). We denote this number as $N$.

An exact solution to this problem constitutes the solution of a combinatorial optimization problem where all combination of cells that constitute a path of length $N$ is evaluated for reduction in uncertainty. Obviously, this is a time-consuming computational process even for values of $N$ that are quite small. For example, $N=10$ would require $6^{10} \cong 6 \times 10^{7}$ evaluations. So, instead of trying to solve this problem exactly, we formulate another problem which is slightly different and then propose a solution that has much less complexity. In order to do this, the requirement of Item 1 above is changed to the following.

1a) Maximize the sum of the uncertainty values associated with the cells that constitute the path $P$ for each sortie.

Note that the problem, as stated in item 1a above, is equivalent to item 1 above if only a single visit is allowed to each cell. Since we do not have any such restriction (as the UAV is allowed to spend more than one time step in a cell, and even revisit a cell), these two requirements, strictly speaking, are not equivalent. However, since we make the assumption that the uncertainty values of a cell is computed only after a sortie, this modification to the problem does not change the solution.

It is relevant to mention here that this problem is also different from the well-known traveling salesman problem [16], where there is no path length constraint and the requirement is to visit all the cells and incur minimum cost.

\section{B. Solution Method}

Given two nodes $S$ (source node) and $T$ (target node) in a graph $G(V, E)$, where $V$ represents the vertices of the graph and $E$ represents the edges or arcs of the graph, we need to find a path $P$ such that it maximizes the sum of the costs associated with the edges constituting the path, where the path is of fixed length $N$.

Let $P=\left(V_{1}, V_{2}, \ldots, V_{N}\right)$, where $V_{1}=S, V_{N}=T$, $V_{i} \in V$, and $V_{i+1} \in \mathcal{N}\left(V_{i}\right)$, where $\mathcal{N}\left(V_{i}\right)$ denotes the neighboring nodes of $V_{i}$, that is, those nodes that are connected with $V_{i}$ with an edge.

Hence, we need to find

$$
\max _{\mathcal{P}} \sum_{i=0}^{n-1} U\left(V_{i}, V_{i+1}\right)
$$

where $U\left(V_{i}, V_{i+1}\right)$ is the uncertainty value associated with the edge connecting the nodes $V_{i}$ and $V_{i+1}$, and $\mathcal{P}$ is the collection of all possible paths of length $N$ from the source node $S$ to the target node $T$.

In principle, this problem can be solved using the combinatorial optimization approach. However, we propose a less complex solution here using some shortest path algorithms available in the literature. An algorithm in the same spirit is Dijkstra's shortest path algorithm finds the shortest path (in terms of minimizing the sum of the weights on the edges of the path) between $S$ and $T$ [16]. However, we cannot use the shortest path algorithms directly since ours is a maximization problem. To transform the problem to a shortest path class of problem, we first convert the problem to a minimization one by replacing the uncertainty values with certainty values. We define $C\left(V_{i}, V_{j}\right)=1-U\left(V_{i}, V_{j}\right)$ as the certainty value associated with an edge between adjacent vertices $V_{i}$ and $V_{j}$ in the graph.

Thus, the new problem formulation is,

$$
\min _{\mathcal{P}} \sum_{i=0}^{n-1} C\left(V_{i}, V_{i+1}\right)
$$

where $C\left(V_{i}, V_{i+1}\right)=1-U\left(V_{i}, V_{i+1}\right)$ is the certainty value associated with the corresponding edge.

Let us try to solve this problem using Dijkstra's shortest path algorithm (assuming, for the sake of argument, that the initial and the final node in the path $P$ are different). Let us also remove the constraint on the number of steps $N$ since Dijkstra's algorithm does not permit such constraints. Suppose $P^{\prime}=\left(V_{1}^{\prime}, \ldots, V_{n^{\prime}}^{\prime}\right)$ be the corresponding shortest path of length $n^{\prime}$. Then the cost associated with path $P^{\prime}$ is

$$
J\left(P^{\prime}\right)=\sum_{i=0}^{n^{\prime}-1} C\left(V_{i}^{\prime}, V_{i+1}^{\prime}\right)=n^{\prime}-\sum_{i=0}^{n^{\prime}-1} U\left(V_{i}^{\prime}, V_{i+1}^{\prime}\right) .
$$

If $n^{\prime}=N$ then this would be the solution to the original maximization problem. But there is no 
guarantee that $n^{\prime}=N$, especially when the source and target (or destination) cells are the same, as assumed in this problem. Thus, a straightforward use of Dijkstra's shortest path algorithm, even when the source and destination cells are different, does not solve the original problem.

In the following, we propose another method, based on the $k$-shortest path algorithm which is an extension of Dijkstra's shortest path algorithm, that yields a satisfactory solution. Assume that the minimization problem as posed above is solved using the $k$-shortest path algorithm $[17,18]$. This algorithm produces paths of monotonically increasing costs starting with the shortest path. Before we describe our approach, in the following, we give a brief description of Dijkstra's shortest path algorithm and the $k$-shortest path algorithm.

\section{Shortest Path Algorithm}

Given a directed graph $G=(V, E)$, where $V$ is the set of nodes or vertices and $E$ is the set of arcs or edges, and a specified source node $s \in V$, where each edge $(i, j) \in E$ has a specified nonnegative length $C_{i j}$, we are required to find, for each node $i \in V$, the shortest path from $s$ to $i$.

Dijkstra's algorithm, described below, creates a a directed shortest path tree $T$ rooted at the source node $s$. We follow the convention that if $(i, j) \in T$ then $\operatorname{pred}(j)=i$, where $i$ is said to be the predecessor of $j$.

\section{Initialization:}

i) Set $S=\varnothing$ and $\bar{S}=V$.

ii) $d(i)=\infty$ (Actually a very large number)

for each node $i \in V$ where $d(i)$ is a label that will ultimately store the distance of the shortest path from $s$ to the node $i$.

iii) $d(s)=0$ and $\operatorname{pred}(s)=0$.

Main loop:

while $|S|<n$, where $n$ is the total number of nodes

let $i \in \bar{S}$ be a node such that $d(i)=\min \{d(j)$ :

$j \in \bar{S}\}$

$S=S \cup i$

$\bar{S}=\bar{S} \backslash i$

for each $(i, j) \in E$

if $d(j)>d(i)+C_{i j}$ then $d(j)=d(i)+C_{i j}$ and $\operatorname{pred}(j)=i$ end

end

\section{The $k$-Shortest Path Algorithm}

The $k$-shortest path problem is to list the $k$ paths, with increasing costs, connecting a given source-destination pair in a directed graph. This problem may be solved using the recursive enumeration algorithm (REA) [18]. The algorithm is recursive, and extends Dijkstra's shortest path algorithm, using Bellman's principle of optimality [16].

The problem is formally stated as follows. Given a directed graph (or digraph) $G=(V, E)$. An edge $(i, j)$ connects nodes $i$ and $j$. As before, each edge $(i, j)$ has a nonnegative length, and it is denoted as $C(i, j)$ and the predecessor set of $j$ is denoted as the set $\operatorname{pred}(j)=\{i:(i, j) \in E\}$. A path $\pi$ between two nodes $u, v \in V$, is defined as a sequence of nodes, $\pi=\pi_{1} \cdot \pi_{2} \cdot \pi_{3} \ldots \ldots \pi_{|\pi|}$, where $\pi_{i} \in V, \pi_{1}=u, \pi_{|\pi|}=v$, and the edges $\left(\pi_{i}, \pi_{i+1}\right) \in E$ for $1 \leq i \leq|\pi|$, where $|\pi|$ is defined as the number of nodes in the path $\pi$. The cost associated with the path $\pi$ is

$$
C(\pi)=\sum_{i=1}^{i=|\pi|} C\left(\pi_{i}, \pi_{i+1}\right)
$$

where $C(\pi)=0$ if $|\pi|=1$. Now, the problem is to compute the $k$-shortest paths from the source node $s \in$ $V$ to a destination node $t \in V$. For every node $v \in V$, the $k$ th shortest path from $s$ to $v$ is denoted by $\pi^{k}(v)$ and the cost associated with it is $C^{k}(v)=C\left(\pi^{k}(v)\right)$. Define $\mathcal{P}^{k}(v)$ as the set of the $k$-shortest paths starting at $s$ and ending at $v$. Note that it contains all the paths starting from the shortest to the $k$ th shortest. This is the set that we want to create.

The problem is solved recursively and in two parts. First, $C^{1}(v)$ (the cost associated with the shortest path from source node to a node $v$ ) and $\pi^{1}(v)$ (the shortest path from source node to a node $v$ ) are computed for all $v$ in $V$ using the shortest path algorithm given above. Next, we use the recursive relation obtained from the generalized Bellman's equation to obtain the cost of the $k$ th shortest path. This recursive equation is given as

$$
\begin{aligned}
& L^{k}(v)= \\
& \begin{cases}0 & \text { if } \quad(k=1) \text { and } \quad(v=s) \\
\min _{u \in \operatorname{pred}(v)} L^{p(u, v, k)}(u)+C(u, v) & \text { otherwise }\end{cases}
\end{aligned}
$$

where

$$
\begin{aligned}
& p(u, v, k)= \\
& \left\{\begin{array}{ll}
1 & \text { if } k=1 \\
p(u, v, k-1)+1 & \text { if } \quad(k>1) \text { and } \\
p(u, v, k-1) & \left(\pi^{k-1}(v)=\pi^{p(u, v, k-1)}(u) \cdot(v)\right)
\end{array} .\right.
\end{aligned}
$$

Note that $p(u, v, k)$ is the number of paths in $\mathcal{P}^{k-1}(v)$ that ends with the edge $(u, v)$, plus one.

Finally, the $k$ th shortest path is obtained from (7) as

$$
\pi^{k}(v)=\pi^{p(\hat{u}, v, k)}(\hat{u}) \cdot v
$$

where

$$
\hat{u}=\arg \min _{u \in \operatorname{pred}(v)} L^{p(u, v, k)}(u) \cdot v .
$$


TABLE I

Results of $k$-Shortest Path Algorithm

\begin{tabular}{ccc}
\hline \hline Path & Length & Cost \\
\hline$P^{1}$ & $n^{1}$ & $J\left(P^{1}\right)=\sum_{i=0}^{n^{1}-1} C\left(V_{i}^{1}, V_{i+1}^{1}\right)$ \\
$P^{2}$ & $n^{2}$ & $J\left(P^{2}\right)=\sum_{i=0}^{n^{2}-1} C\left(V_{i}^{2}, V_{i+1}^{2}\right)$ \\
$\vdots$ & $\vdots$ & $\vdots$ \\
$P^{k}$ & $n^{k}$ & $J\left(P^{k}\right)=\sum_{i=0}^{n^{k}-1} C\left(V_{i}^{k}, V_{i+1}^{k}\right)$ \\
\hline
\end{tabular}

\section{E. Search Route Computation}

Using the above two algorithms we can compute and rank order the shortest paths of increasing sum of certainty values for paths starting from and ending at the same node (which, in this case, represents the base station of the UAVs). The results may be tabulated as shown in Table I. Note that the length of a path is the number of steps in the path (and represents the flight time of the vehicle) and the cost is the sum of the certainty values associated with the cells constituting the path.

In this table, the path $P^{1}$ is the shortest path, followed by paths of increasing costs, so that $J\left(P^{j+1}\right) \geq J\left(P^{j}\right)$. In this table, consider the first path, as we go down the table, that has a length of $N$ (that is, a path of $N$ steps). We claim that this path is the optimal path for the original maximization problem. This path is also the path with the minimum cost among all paths of length $N$. Note that there could be several such paths which have the same length $N$ and satisfy the minimum cost criterion. However, we may select any one of them.

To prove our claim, let this path be the $j$ th one in the above sequence. Then,

$$
J\left(P^{j}\right) \leq J\left(P^{j+s}\right), \quad s=1,2, \ldots, l
$$

where $P^{j+l}$ is the last path in this sequence which has length $N$. Then,

$$
\begin{aligned}
\sum_{i=0}^{n-1} C\left(V_{i}^{j}, V_{i+1}^{j}\right) \leq \sum_{i=0}^{n-1} C\left(V_{i}^{j+s}, V_{i+1}^{j+s}\right), \quad s=1, \ldots, l \\
\Rightarrow \sum_{i=0}^{n-1} U\left(V_{i}^{j}, V_{i+1}^{j}\right) \geq \sum_{i=0}^{n-1} U\left(V_{i}^{j+s}, V_{i+1}^{j+s}\right), \quad s=1, \ldots, l
\end{aligned}
$$

which implies that $P^{j}$ is the solution to the original problem.

Hence, by using the $k$-shortest path algorithm we are able to solve the original problem. It may so happen that there are no paths of length $N$ that figure in this table. In that case, a solution to the problem does not exist. This can happen, for example, when the specified path length is too small to allow the UAV to get from $S$ to $T$. Or else, it can happen if $S$ and $T$ are the same (as in our case) and the specified value of $N$ is an odd number. However, these are pathological cases and do not detract from the utility of the algorithm.

We see in the simulations that when $S$ and $T$ are the same (that is, UAVs fly from their bases and return to the same bases), it retraces the path back during its return. This retracing of paths happens because of the fact that we do not update the uncertainty maps till the UAV comes back to the base station. So, the fact that an UAV has already passed over a cell does not change its uncertainty until the UAV is still searching. As a result, the return path is exactly the same as the forward path, since this yields the optimal path. If we relax this assumption, then we have to cope with the uncertainty values changing dynamically with the path taken by a UAV until that step. This will definitely improve the search performance of the UAVs. However, implementing this feature requires a nontrivial modification to the $k$-shortest path algorithm. Efficient algorithms to obtain $k$-shortest paths where the edge costs change dynamically as a function of the path itself, are still not available in the literature. The only algorithm that can be designed is combinatorial and has a complexity of much higher order than that which can be handled by our computational resources even for a reasonable sized problem. Development of such algorithms is still in the research domain and is beyond the scope of the present paper.

\section{APPLICATION TO MULTIPLE UAVS CASE}

Normally, teams of UAVs are used for search and surveillance task. The algorithm given above produces the required optimal path for a single searcher. However, this algorithm can be easily used for multiple UAVs that carry out this task in a cooperative fashion as described in [6]. In fact, preliminary results obtained for the multiple UAVs case has shown that even very simple search strategies can effectively improve the search performance of multiple UAVs [19].

It is assumed that the initial uncertainty map is available to all the UAVs (which may be located at a single base or at different bases in the search region). Each UAV would then determine the optimal route for its first sortie using the search algorithm. At the end of the sortie, the base stations cooperatively exchange the data collected by individual UAVs and use the collective data to together update the uncertainty map for the next sortie. If the information content (or data) of all the base stations are the same (which would happen when there exists a fully 


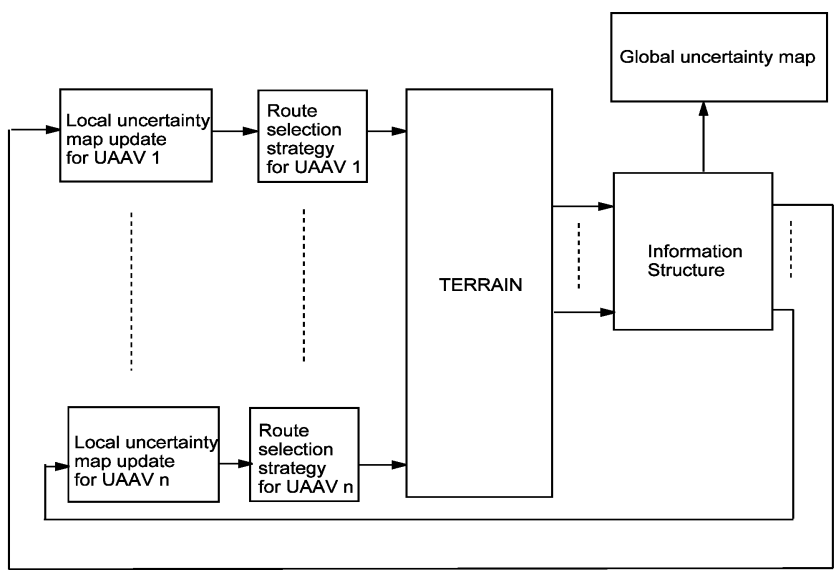

Fig. 4. Uncertainty map update and route selection.

connected communication network, with noiseless communication, between all the base stations), then all the UAVs implement the algorithm using the same uncertainty map. On the other hand, if there is only partial connectivity or noisy communication, the UAVs may have different uncertainty maps at each stage. In the following, we explore some of these possibilities and illustrate them with simulation results.

Note that the algorithm for finding the search routes does not change in any of the following cases. It is only the uncertainty map used by the searchers that is different for different information structures. Hence we need to describe the information based on which the uncertainty map updating is done by each searcher. For this we define some notations below.

Let $\rho_{k}^{i}$ denote the search route followed by searcher $i$ during its $k$ th sortie. Note that a search route is a sequence of cells that the searcher has passed through. Let the uncertainty map of searcher $i$ at the beginning of sortie $k$ be denoted by $\mathcal{U}_{k}^{i}$. In other words, $\rho_{k}^{i}$ is determined, using the search algorithm, based upon the uncertainty map $\mathcal{U}_{k}^{i}$.

The block diagram given in Fig. 4 shows the sequence in which the uncertainty map update and the route selection is done. Each UAV uses its locally updated uncertainty map to generate its own search routes. The information structure block decides the information that is available to each UAV based on connectivity and information latency. Thus, the locally updated uncertainty map could be different for different UAVs. The global uncertainty map is the one that will be obtained if all the UAVs exchange information among themselves. Below we consider a few possible information structures and the consequent update formula for the local uncertainty maps after each sortie.

\section{A. Perfect Information Case}

In this case, each searcher communicates its search route after a sortie to all the other searchers. Hence,

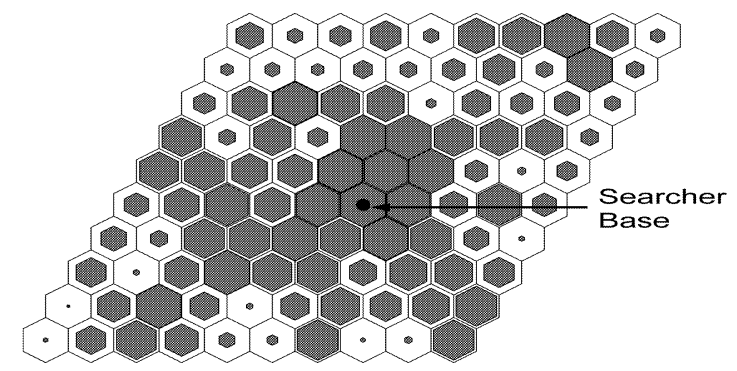

Fig. 5. Initial uncertainty map.

the updating of the uncertainty map by each searcher results in the same uncertainty map, provided that they all started with the same initial uncertainty map. Thus,

$$
\mathcal{U}_{k}^{i}=f\left(\mathcal{U}_{k-1}^{i} ; \rho_{k-1}^{1}, \rho_{k-1}^{2}, \ldots, \rho_{k-1}^{n}\right) .
$$

If $\mathcal{U}_{0}^{1}=\mathcal{U}_{0}^{2}=\cdots=\mathcal{U}_{0}^{n}=\mathcal{U}_{0}$ (say) then,

$$
\mathcal{U}_{k}^{1}=\mathcal{U}_{k}^{2}=\cdots=\mathcal{U}_{k}^{n}
$$

for all sorties $k$, and is denoted by $\mathcal{U}_{k}$.

\section{B. Partially Connected Case}

In this case each searcher receives information from only a subset of the searchers. This is the buddy set concept proposed in [20]. Define $N^{i}$ as the set of searchers who send their information to searcher $i$. Then the uncertainty map is updated as follows:

$$
\mathcal{U}_{k}^{i}=f\left(\mathcal{U}_{k-1}^{i} ; \rho_{k-1}^{j} \mid j \in N^{i}\right) .
$$

For compactness of notation, we assume that $i \in N^{i}$.

\section{Delayed Information Case}

In this case each searcher receives information from all the searchers, but with a time delay of $d$ steps. The delay is measured in terms of the number of sorties so that $d=2$ would imply that a searcher on its $k$ th sortie will have information about the search route of the other searchers on their $k-3$ rd sortie, and no information about these searchers' route on their $k-2$ th and $k-1$ th sortie. In general,

$$
\begin{array}{r}
\mathcal{U}_{k}^{i}=f\left(\mathcal{U}_{k-1}^{i} ; \rho_{k-1}^{i}\right), \quad \text { for } \quad k=1, \ldots, d+1 \\
\mathcal{U}_{k}^{i}=f\left(\mathcal{U}_{k-1}^{i} ; \rho_{k-d+1}^{1}, \ldots, \rho_{k-d+1}^{i-1}, \rho_{k-1}^{i}, \rho_{k-d+1}^{i+1}, \rho_{k-d+1}^{n}\right), \\
\text { for } \quad k>d+1 .
\end{array}
$$

So, a searcher's own information only is up to date and all others are delayed. This may be further generalized in an obvious way to account for different delays for each searcher. We omit details. 

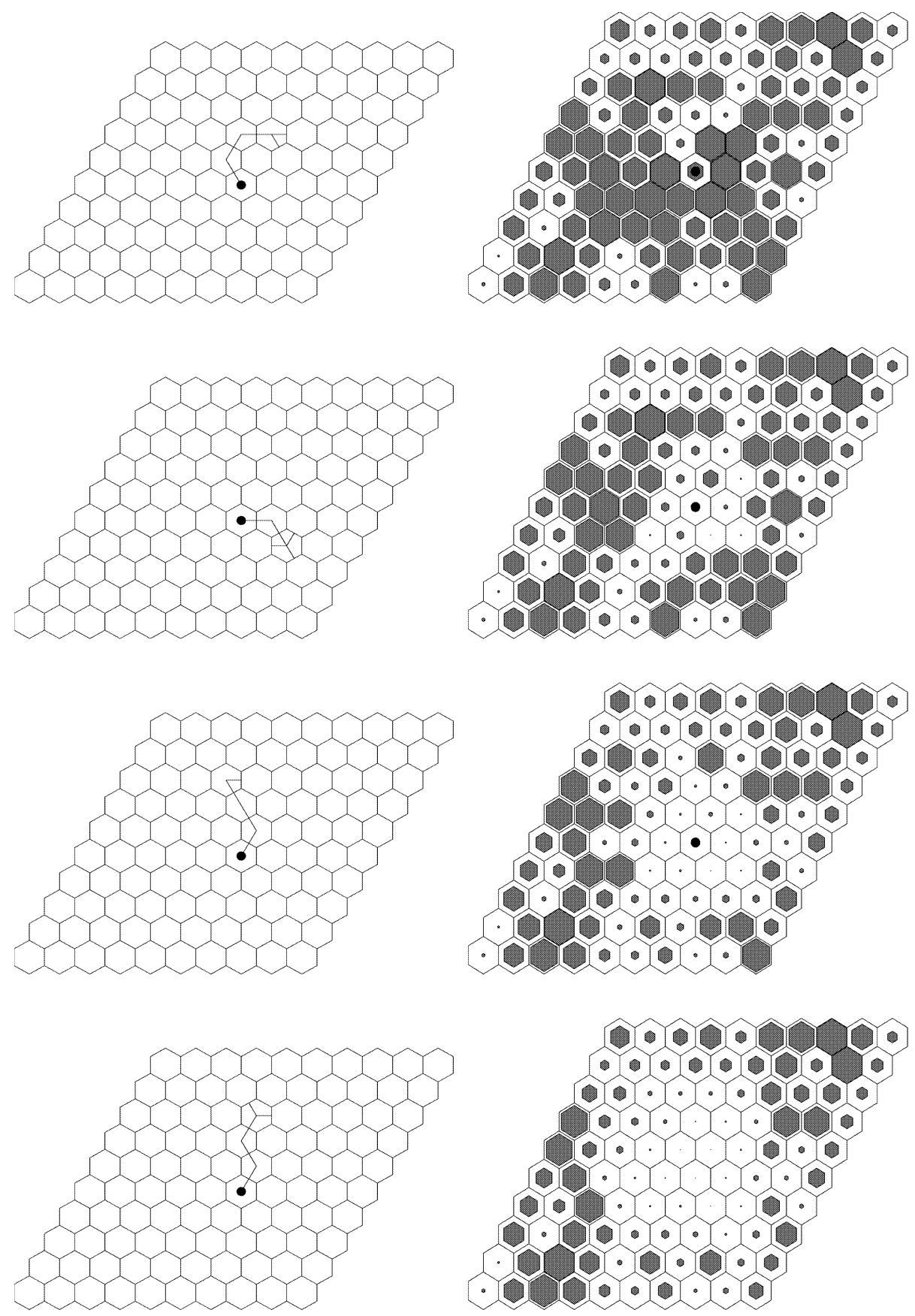

Fig. 6. Single searcher; $k$-shortest path strategy, sorties $1,6,12$, and 18 .

D. Partially Connected with Delayed Information

In this case, each searcher receives information with a delay $d$ but only from a subset of the searchers. So,

$$
\begin{aligned}
& \mathcal{U}_{k}^{i}=f\left(\mathcal{U}_{k-1}^{i} ; \rho_{k-1}^{i}\right), \quad \text { for } \quad k=1, \ldots, d+1 \\
& \mathcal{U}_{k}^{i}=f\left(\mathcal{U}_{k-1}^{i} ; \rho_{k-1}^{i}, \rho_{k-d+1}^{j} \mid j \in N^{i}\right), \quad \text { for } \quad k>d+1 .
\end{aligned}
$$

As in the earlier case, a generalization in terms of different delays for different searchers is easily modeled in this framework.

\section{SIMULATION RESULTS}

\section{A. Single Searcher}

In the single searcher case, only the perfect information case is relevant. For the purpose of simulation we consider a region composed of a $10 \times 10$ grid of hexagonal cells. The initial uncertainty map is created by generating random numbers between 0 and 100 (thus representing uncertainty as a percentage). This is shown in Fig. 5.

The uncertainty is shown as the grey area within the cell. The percentage of uncertainty in a cell is 

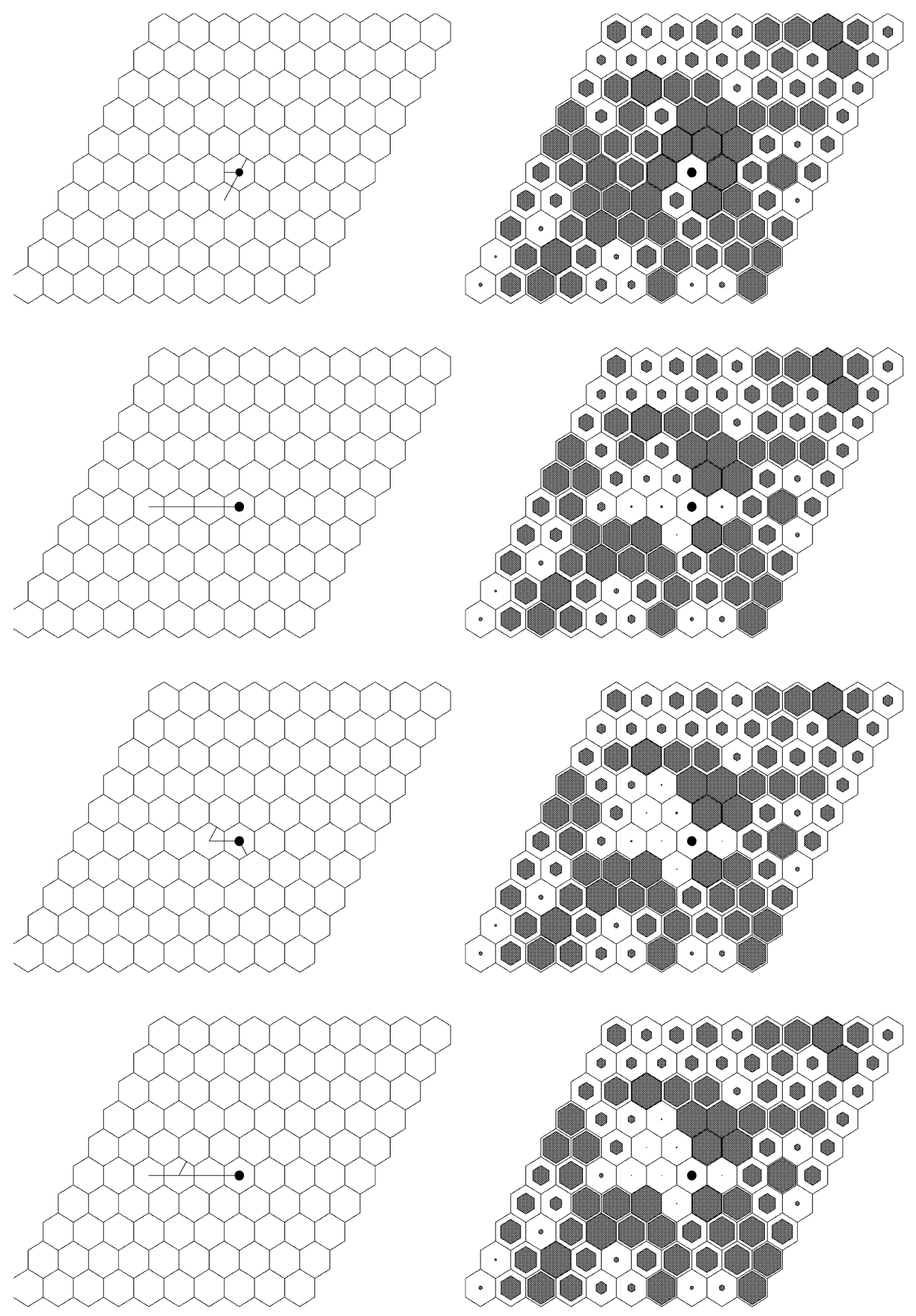

Fig. 7. Single searcher, random search strategy, sorties 1, 6, 12, and 18 .

proportional to the size of the grey area in the cell. The position of the base station is marked with a $\bullet$ in the figure. First, we consider a single UAV that flies a total of 18 sorties through this region. Each sortie is for a limited length of 16 steps through the graphical model, which means that the UAV flies for 8 steps in the hexagonal grid, which in turn implies that it can reach only 4 cells deep from its base station since it has to come back to the base after a sortie.

In the following, we present the simulation results for some intermediate steps in the search operation. The details of the search operation for each sortie is available in [21]. We show only a few intermediate steps here for brevity.

Using the algorithm proposed in this work, Fig. 6 shows sortie $1,6,12$, and 18 of the UAV and the consequent change in the uncertainty map. The uncertainty map is updated using (2) and (14), for the single searcher case. Note that the shrinking of the grey area in the cells with increasing number of sorties denotes the reduction in uncertainty about that cell.

These figures give a visual representation of how the uncertainties reduce with increasing number of sorties. In each of these figures the figure on the left 

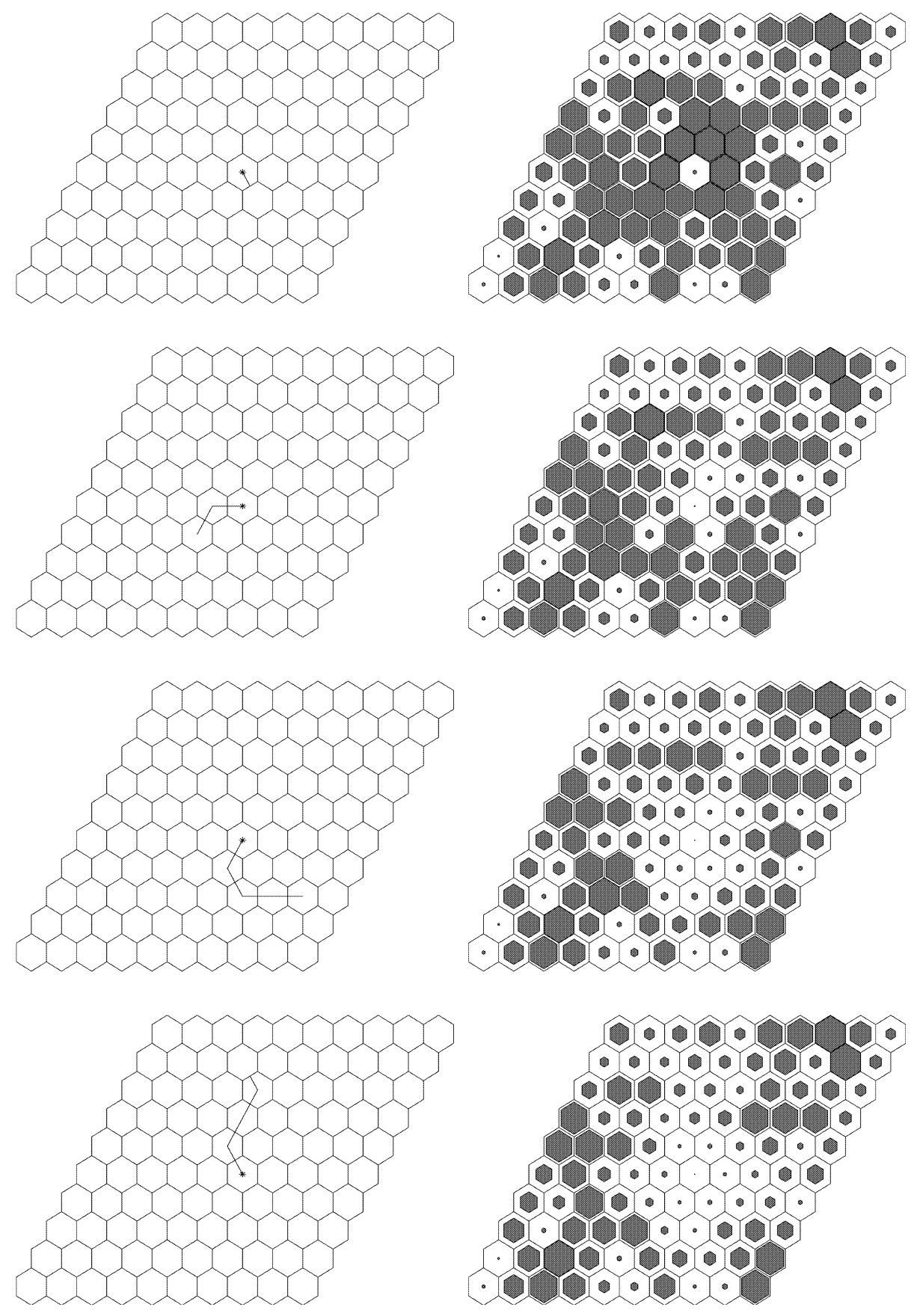

Fig. 8. Single searcher, greedy search strategy, sorties $1,6,12$, and 18 .

shows the UAV route as computed by the $k$-shortest path technique, and the figure on the right shows the updated uncertainty map after this sortie by the UAV. Note that, in several cases, the routes shown for the UAV shows as if the UAV goes up to the boundary of a cell and comes back again. This is actually the representation of the route as obtained from the graphical model. In reality, these path segments should be interpreted to mean that the UAV remains in the cell for more than one time step duration.

To illustrate the efficacy of our algorithm, we compare its performance with a random search strategy and a greedy search strategy. In the random strategy, the UAV selects its next cell for search by generating a uniformly distributed random number for the initial 4 steps on the hexagonal grid, and then it retraces its path back to the base. The routes and the corresponding uncertainty maps for the random search strategy are shown in Fig. 7 for the same sortie numbers as above.

In the greedy search strategy, the UAV selects the next cell in its path by choosing the neighboring cell with the maximum uncertainty. This is done for the initial 4 steps on the hexagonal grid, and then the 


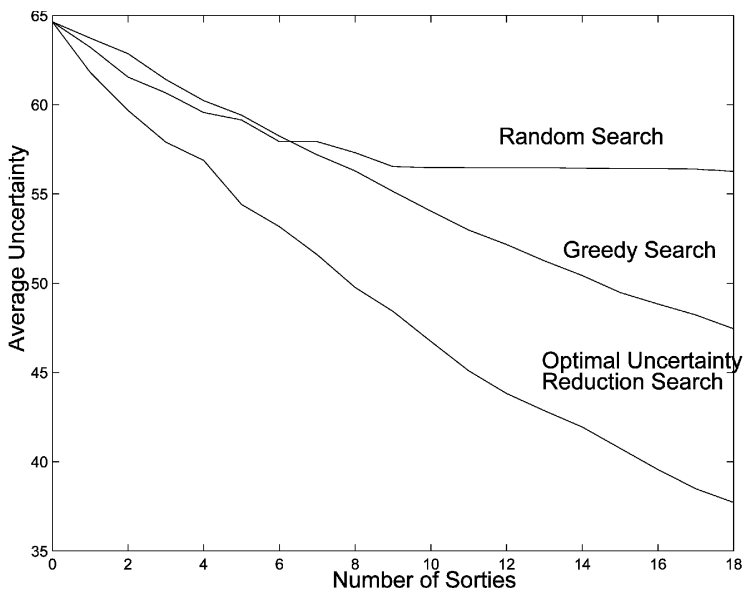

Fig. 9. Reduction in average uncertainty.

UAV retraces its path back to the base. The routes and the corresponding uncertainty maps for the random search strategy are shown in Fig. 8 for the same sortie numbers as before.

In Fig. 9 we show the reduction in average percentage uncertainty value for the search region with increasing number of sorties for the three search algorithms. It clearly shows the superiority of the $k$-shortest path algorithm based strategy over the other less sophisticated algorithms.

\section{B. Multiple Searchers}

Finally, we apply our search algorithm to multiple UAVs with perfect information. We use the same

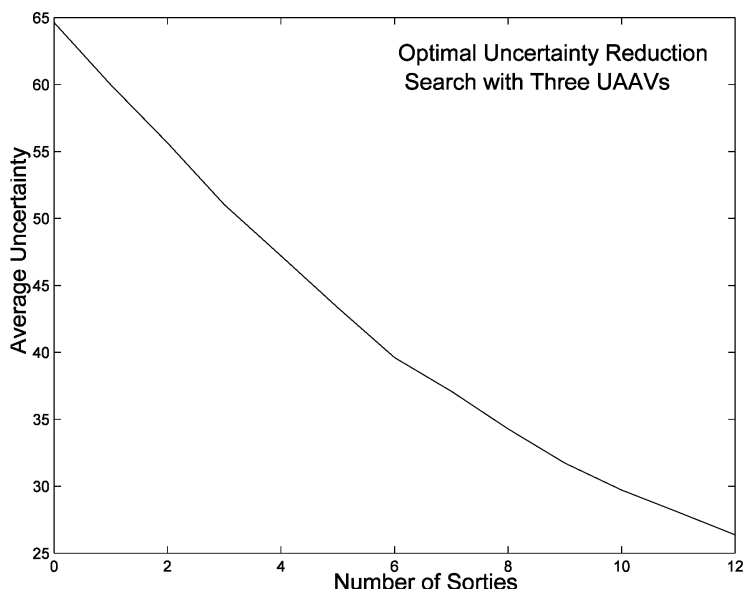

Fig. 11. Reduction in average uncertainty for three UAVs case.

search region as before, but assume that the search is being conducted by three UAVs. All the searchers use the same algorithm and cooperatively update their uncertainty maps based on information received from the other UAVs at the end of each sortie. The simulations are done for a total of 12 sorties each by the $3 \mathrm{UAVs}$, each of which is assumed to have an endurance of 12 steps in the graph model (which implies an endurance of 6 steps in the hexagonal grids, and in turn implies that each UAV can search 3 cells deep from its base station). The uncertainty maps after 3, 6, 9, and 12 sorties are shown in Fig. 10. The $\bullet$ indicates the positions of the base stations. In Fig. 11 we show the reduction in average uncertainty with increasing number of sorties by the three UAVs.

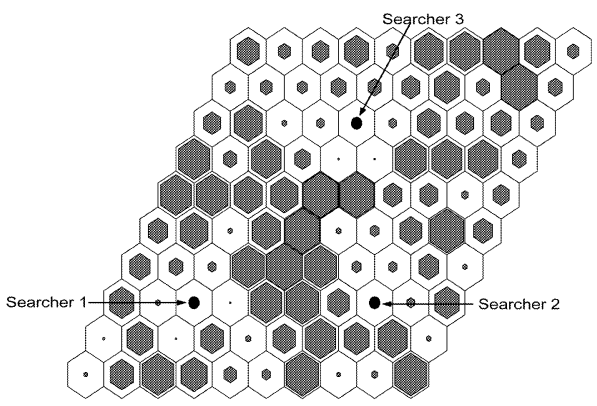

(a)

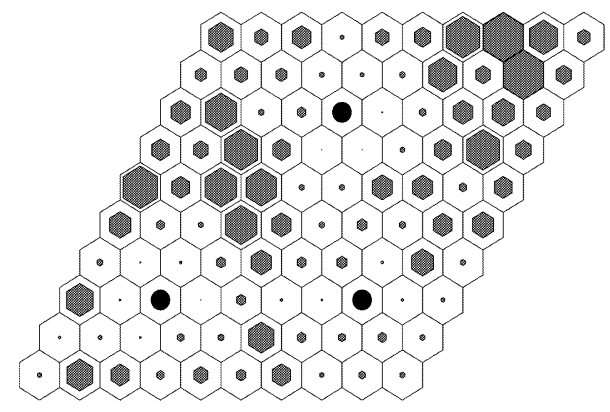

(c)

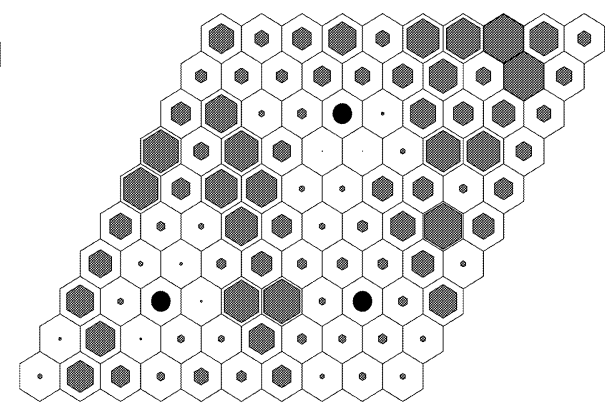

(b)

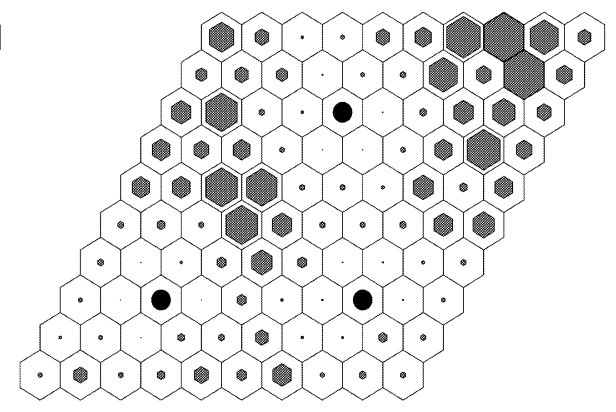

(d)

Fig. 10. Uncertainty map for three UAVs after (a) 3 sorties, (b) 6 sorties, (c) 9 sorties, (d) 12 sorties. Perfect information; $k$-shortest path strategy. 


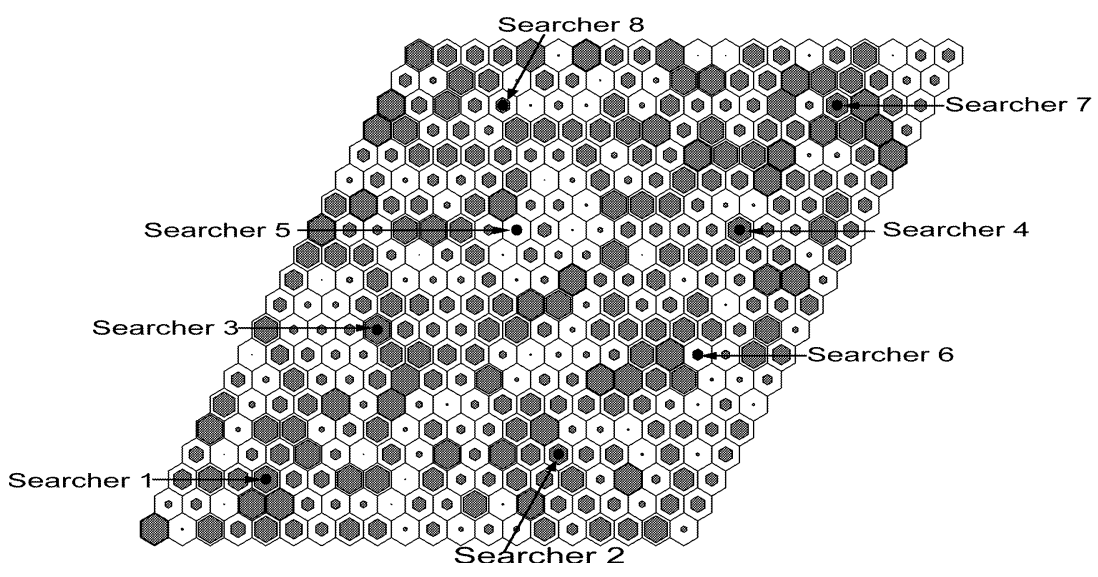

Fig. 12. Initial uncertainty map for 8 searchers case.
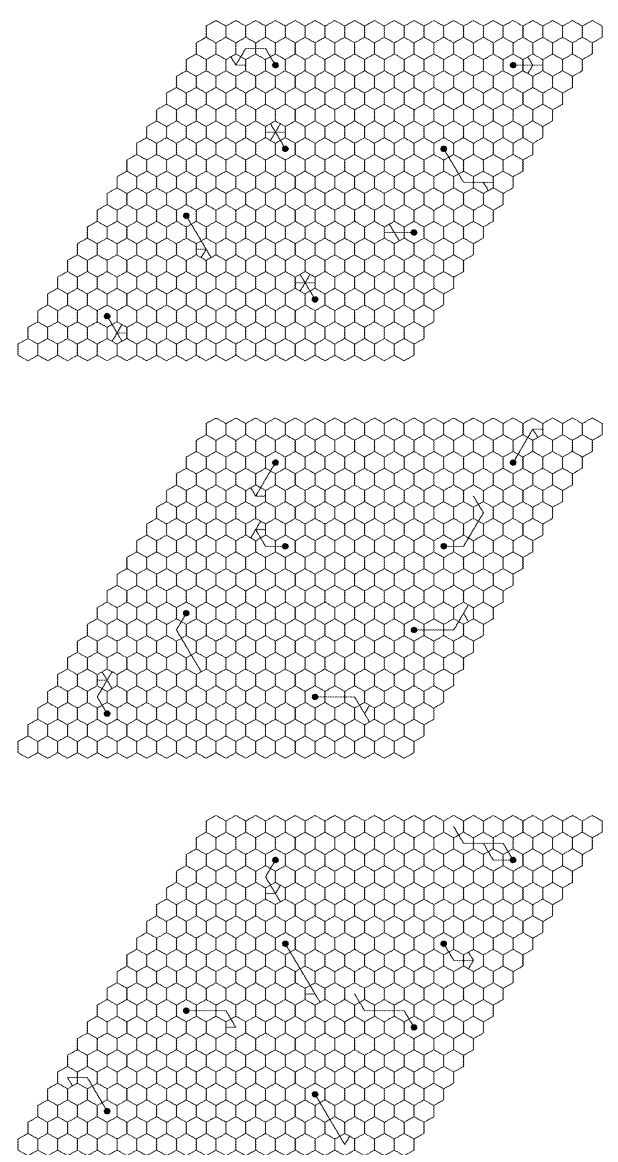
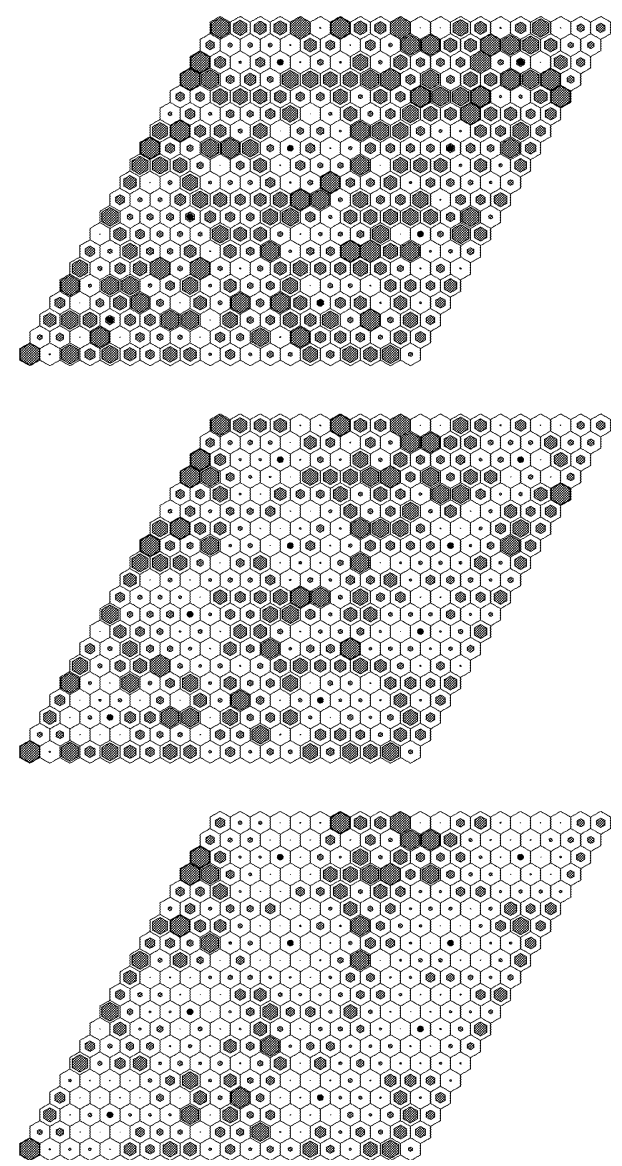

Fig. 13. Eight searchers, perfect information, $k$-shortest path strategy, sorties 1,5 , and 10.

However, to demonstrate the benefit of a group of UAVs carrying out a search operation under various types of information structures and using the algorithm proposed in this report, we consider a large $20 \times 20$ grid as the search region with the initial uncertainty map as shown in Fig. 12. As before, the initial searcher positions are marked with a $\bullet$. Each searcher is assumed to have an endurance equivalent of 16 steps in the graph model which translates to a search of maximum depth 4 in the grid model of the search region. A total of 10 sorties for each searcher is considered in this model.

We carry out several simulations. In the first set of simulations, we assume the searchers to be fully connected with each other through wireless communication links and thus all of them select their search strategies based upon the same uncertainty map at each sortie. This is the perfect information or the complete information case, where the global information about the uncertainty map is available to 

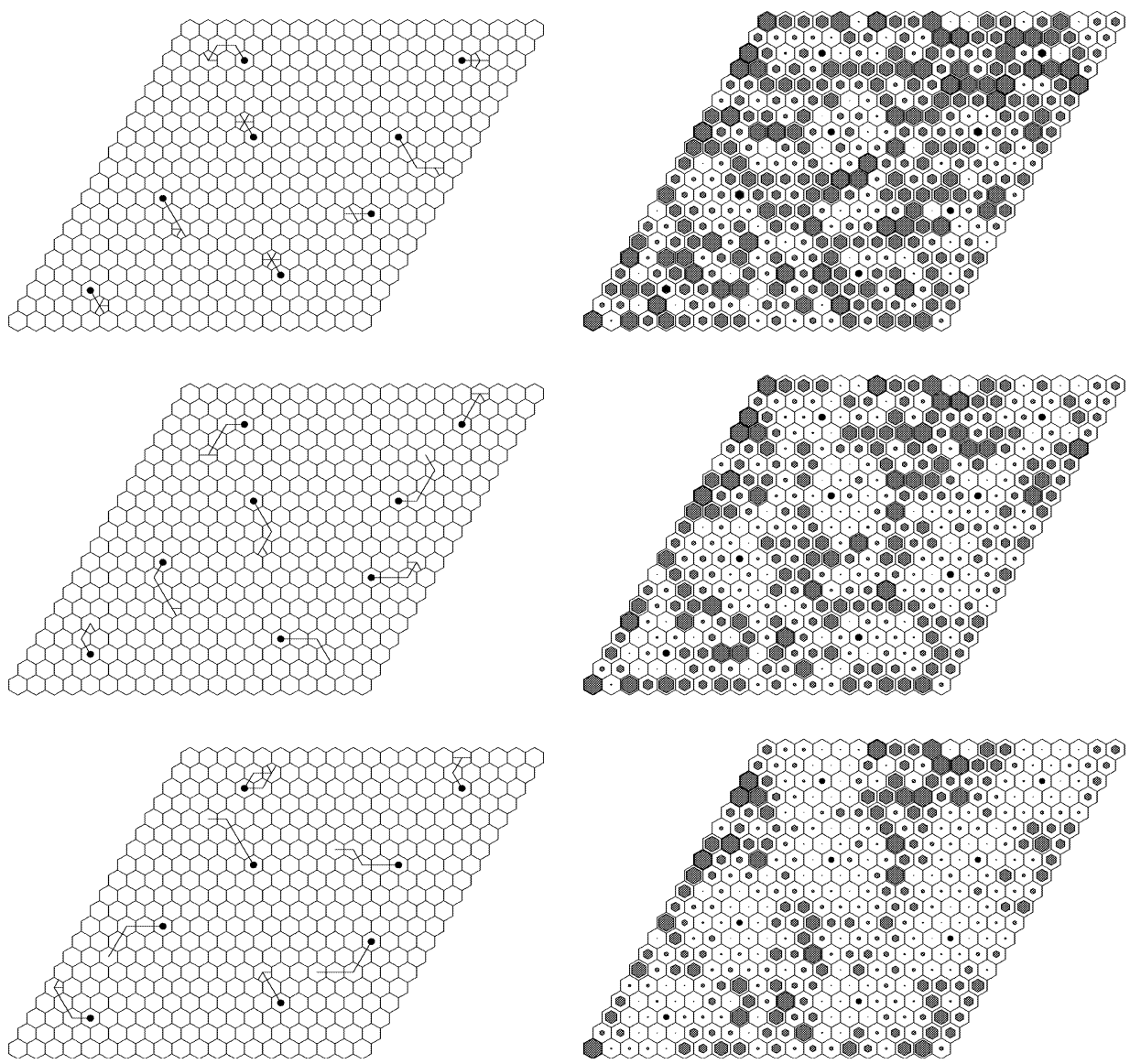

Fig. 14. Eight searchers, delayed information, $k$-shortest path strategy, sorties 1, 5, and 10.
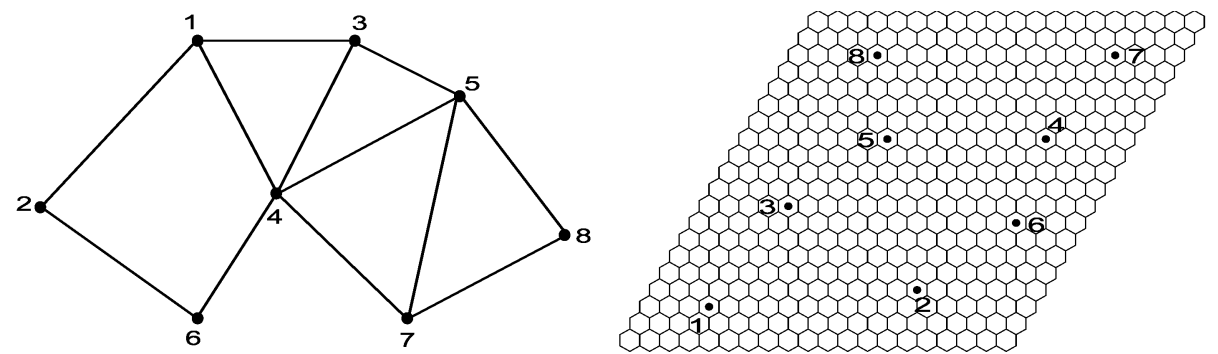

Fig. 15. Network connectivity for partial information case and location of the searchers.

each searcher. This is also the same as a centralized model. Fig. 13 shows the search routes for the 1st, 5 th, and 10th sortie, and the resulting uncertainty map after each sortie. The uncertainty maps are updated using (14) and (2).

In the next set of simulations, we consider a delayed information case in which searchers receive information from other searchers but with a delay of one step length. Which means that a searcher updates its uncertainty map just before the $k$ th sortie, based on its own search route in the previous sortie $k-1$ and the search routes of the other searchers in the sortie $k-2$. As a result, the uncertainty map of each searcher is different. In Fig. 14 we show the search routes for all the 8 searchers, for the $1 \mathrm{st}, 5$ th, and the 10th sorties, and the corresponding global uncertainty map, which can be looked upon as the uncertainty map that will be obtained if the UAVs stop searching at that sortie and then communicate all the information to a central commander which will then be able to create this global uncertainty map using all the current information. Note that these uncertainty maps are obtained using (14) and (2). However, the uncertainty maps used by the individual searchers are obtained using (17), (18), and (2), and are different for different searchers.

In the next set of simulations we consider the partial information case in which the wireless communication between searchers is restricted only to subsets of the searchers. Fig. 15 shows the network 

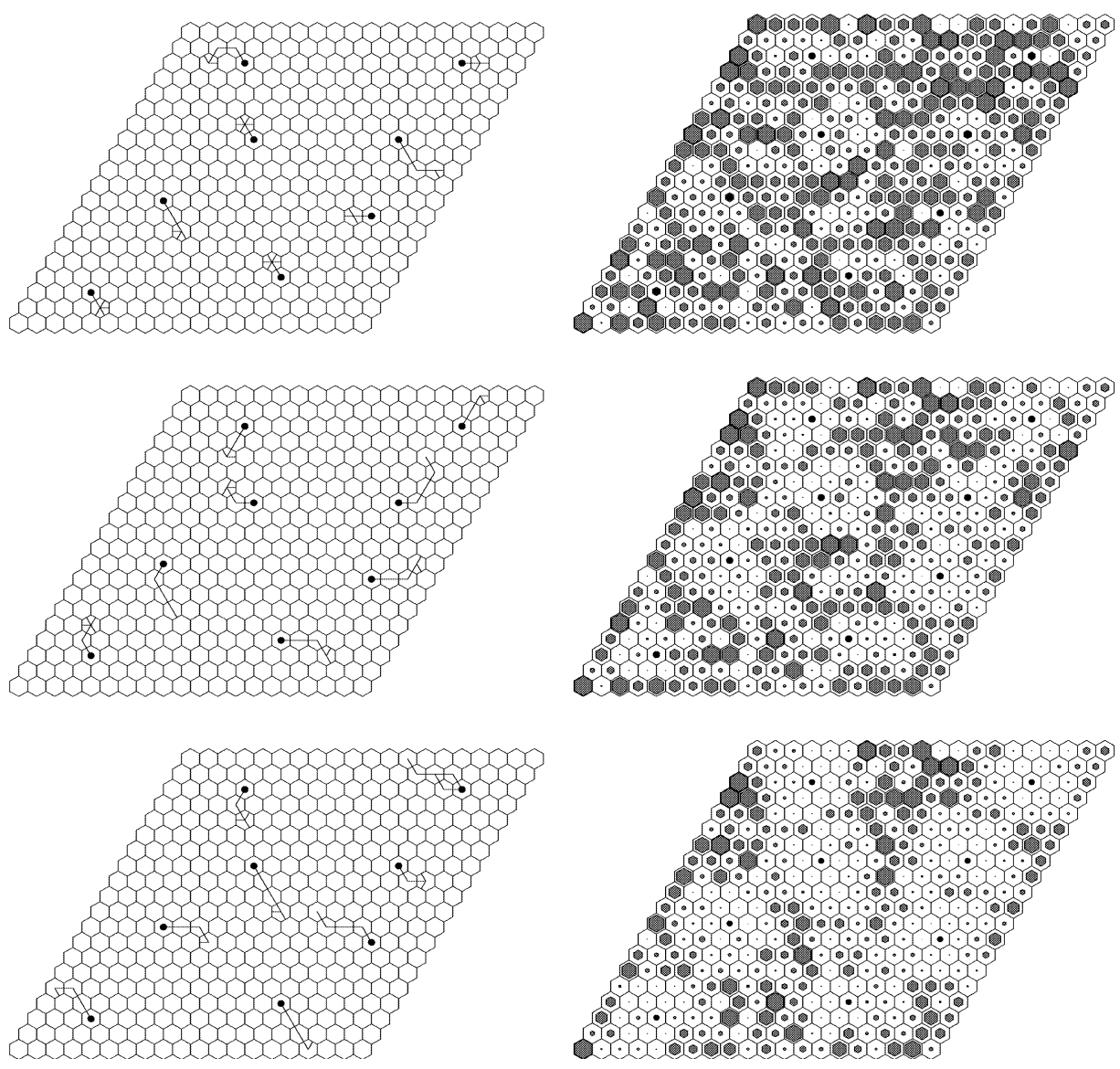

Fig. 16. Eight searchers, partial information, $k$-shortest path strategy, sorties 1,5 , and 10 .

connectivity between searchers and their specific location on the grid search region.

Note that the locations of the searchers are the same as before, but since the network is no longer fully connected, it is necessary to identify the searchers individually. In this case, there is no information delay between searchers, but since each searcher gets information from only a subset of the other searchers, its uncertainty map is updated based only on this limited information. As a result each searcher has a different uncertainty map at the beginning of each sortie (except the first one, since all the initial uncertainty maps are the same). Fig. 16 shows the search routes and the global uncertainty map for the same 1st, 5th, and the 10th sortie. The uncertainty maps used by the individual searchers are obtained using (16) and (2), and are again different for different searchers.

In the next set of simulations, we consider a combination of the delayed information and partial information case. Here, we have the same network connectivity as given in Fig. 15 but the information that a searcher receives from its neighbor is delayed by one step. Hence, here also, the searchers have different uncertainty map at all sorties except the first one. The search routes obtained and the global uncertainty map for this information structure are shown in Fig. 17 for sorties 1, 5, and 10. The uncertainty maps used by the individual searchers are obtained using (19), (20), and (2), and are different for different searchers.

To compare the performance of the search algorithm under different information structures and network connectivity, we plot the average uncertainty of the search region in Fig. 18. The first plot shows that there is not much difference in the average global uncertainty for different information structures. The second plot magnifies a small portion of the original plot to show that the partial information case (with incomplete connectivity) has the same performance as the perfect information case. Although this may look counter-intuitive, the reason behind this lies in the physics of the problem. From Fig. 15 we can observe that the searchers' locations are fairly well separated and since each searcher can search 4 cells deep, they very rarely encroach upon each other's search regions. This means that routes of the other searchers which are far away from a given searcher do not affect the optimal decision of this searcher. The only searchers that affect a given searcher's optimal route are its own immediate geographical neighbors. Now, in this problem the network connectivity is such that the close geographical neighbors are also the ones that are connected by a communication link. So, a searcher 

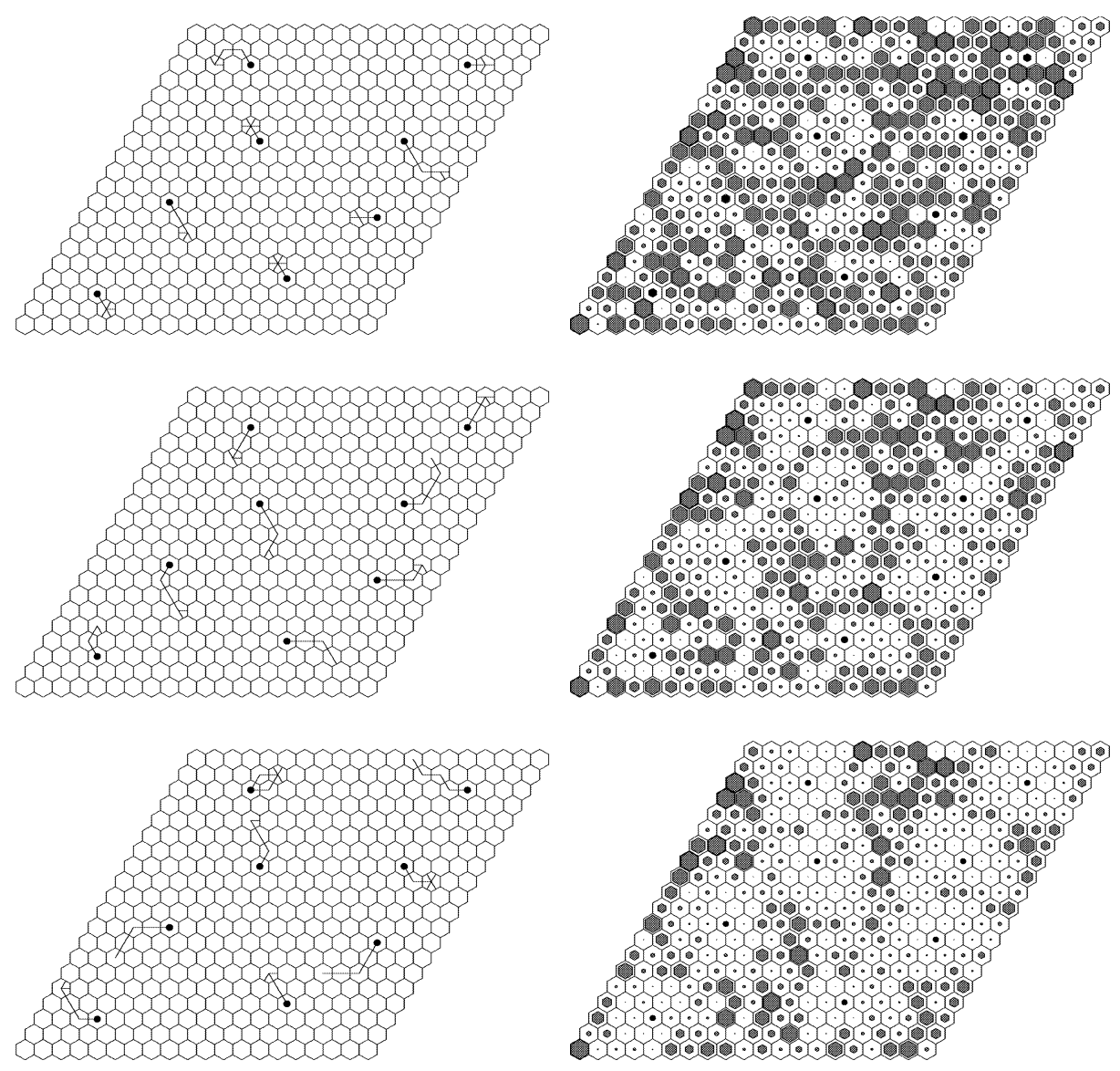

Fig. 17. Eight searchers, partial and delayed information, $k$-shortest path strategy, sorties 1,5 , and 10.
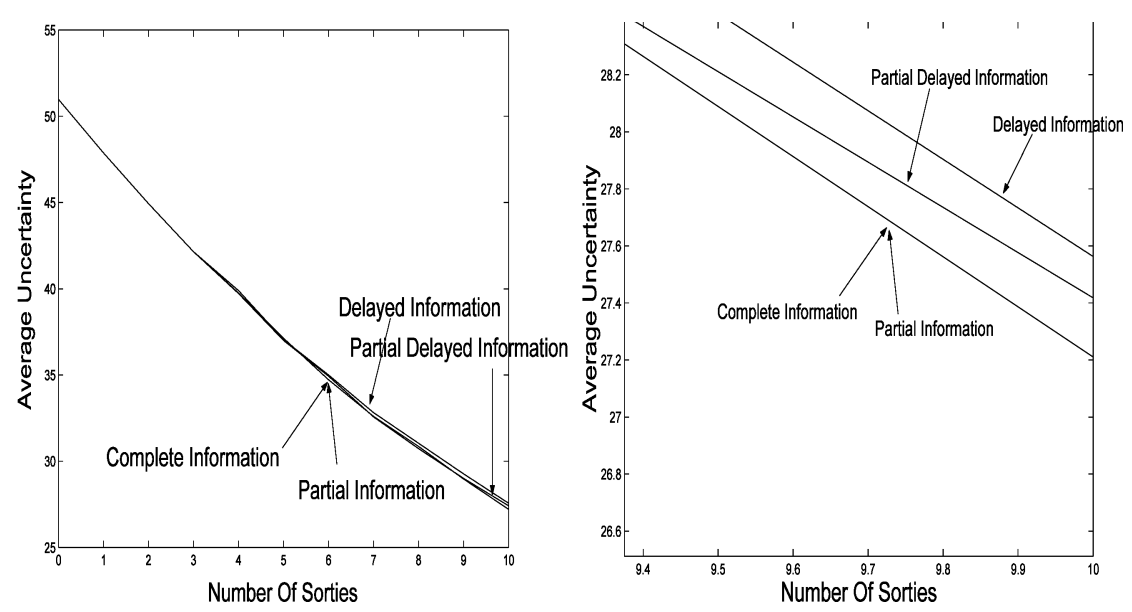

Fig. 18. Comparison of average uncertainties for different information structures.

receives all the relevant information that it needs from its neighbors in spite of incomplete connectivity. Thus, the performance of the partial information case is almost identical to the global information case. On the other hand, when we consider delayed information, it affects the uncertainty map of a searcher as it does not have the current information of its neighbors. This is the reason for its slightly worse performance.

However, the fact remains that since the searcher locations are well separated the partial information and incomplete network connectivity do not affect the search performance much. In the next set of simulations we consider the same problem but with the searchers distributed in a uniform manner around a central position.

Fig. 19 shows the network connectivity between searchers and their specific location on the grid search region. The network connectivity is the same as before, but the locations of the searchers have changed. 

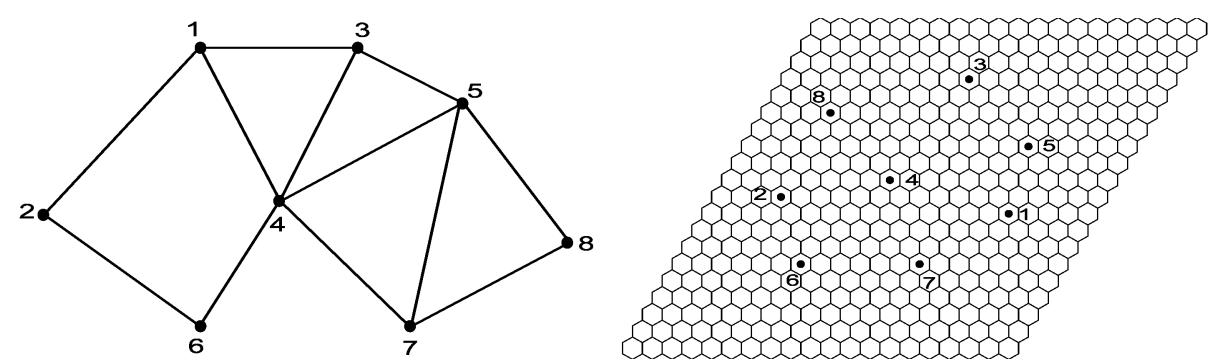

Fig. 19. Network connectivity for partial information case and uniformly distributed location of searchers.
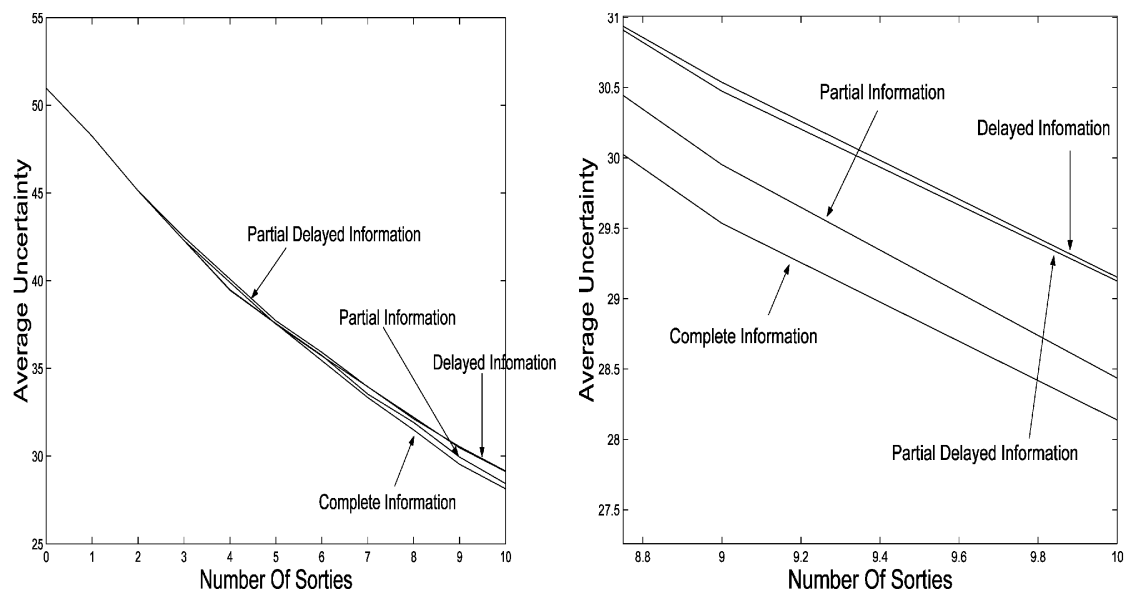

Fig. 20. Comparison of average uncertainties for different information structures for uniform searcher location case.

The initial uncertainty map is the same as before, and each searcher is assumed to have an endurance equivalent of 16 steps in the graph model or a search of maximum depth 4 in the grid model. A total of 10 sorties for each searcher is considered. We omit the detailed uncertainty maps and search routes (these are available in [21]).

To compare the performance we plot the average uncertainty of the search region in Fig. 20. The first plot shows that there is some difference in the average global uncertainty for different information structures. The second plot magnifies a small area in the original plot.

Finally, it should be noted that the algorithm for finding optimal search routes is applicable irrespective of how the searchers are placed. In the simulation examples with multiple searchers, some of the searcher bases are placed close enough to make a slight overlap of their search regions possible. However, we would like to point out that the cooperation between multiple vehicles is limited to using a common uncertainty map, based upon collective information of the previous stage. In fact, we have explored different types and levels of cooperation that arise out of this information exchange scenario in terms of network connectivity and delayed information. As mentioned earlier, we do not address cooperation in terms of real-time exchange of information between UAVs during the search sortie, but rather we assume cooperation between bases that collect information obtained by the UAVs after the end of a sortie, process them, and then collectively update the uncertainty map. Since we do not consider the scenario where cooperation between UAVs is extended to the point where the paths are decided in a cooperative fashion, a closer placement of the UAV bases will not yield any result more insightful than the ones already presented.

\section{CONCLUSIONS}

We proposed a search algorithm based on the well-known $k$-shortest path algorithms used in network flow applications, for use in UAVs searching for targets in unknown environments. The results, compared with standard random search and greedy strategies, show the superiority of the proposed algorithm. Its utility in a cooperative search using multiple UAVs was also demonstrated. We also consider the possibility of delays in information communication and partial network connectivity between searchers as proposed in [20]. An aspect that needs to be reconsidered is the assumption of the uncertainty map remaining unchanged during a sortie. Because of this assumption, the search path is optimal in terms of maximizing the total uncertainty in a path, but is suboptimal in terms of maximum reduction in uncertainty in a given path. However, this would 
require a nontrivial modification of the $k$-shortest path algorithm to a case when the edge weights change as a function of the number of times that the searcher passes through that edge. All these will be considered in later studies.

\section{ACKNOWLEDGMENTS}

The authors would like to acknowledge helpful comments received from D. Eppstein and A. Marzal on the use of their $k$-shortest path algorithm.

\section{REFERENCES}

[1] Benkoski, S. J., Monticino, M. G., and Weisinger, J. R. (1991)

A survey of the search theory literature.

Naval Research Logistics, 38, 4 (Aug. 1991), 469-494.

[2] In Proceedings of the JFACC Symposium on Advances in Enterprise Control, Minneapolis, MN, 2000.

[3] Enns, D., Bugajski, D., and Pratt, S. (2002) Guidance and control for cooperative search. In Proceedings of the American Control Conference, Anchorage, AK, 2002.

[4] McLain, T. W., Chandler, P. R., and Pachter, M. (2002) A decomposition strategy for optimal coordination of unmanned air vehicles.

In Proceedings of the American Control Conference, Chicago, IL, 2002, 369-373.

[5] Schumacher, C., Chandler, P. R., and Rasmussen, S. R. (2002)

Task allocation for wide area search munitions. In Proceedings of the American Control Conference, Anchorage, AK, 2002, 1917-1922.

[6] Passino, K., Ploycarpou, M., Jacques, D., Pachter, M., Liu, Y., Yang, Y., Flint, M., and Baum, M. (2000) Cooperative control for autonomous air vehicles. In Proceedings of the Cooperative Control and Optimization Workshop, FL, Dec. 2000.

[7] Lida, K. (1992)

Studies on the Optimal Search Plan. Lecture Notes in Statistics: 70, Berlin, Springer-Verlag, 1992.

[8] Koopman, B. O. (1980) Search and Screening (2nd ed.). New York: Pergamon, 1980.

[9] Stone, L. D. (1975)

Theory of Optimal Search. New York: Academic Press, 1975.

[10] Goldsmith, S. Y., and Robinett, R. (1998)

Collective search by mobile robots using alpha-beta coordination.

In A. Dragoul, M. Tambe, and T. Fukuda (Eds.), Collective Robotics (Lecture Notes in Artificial Intelligence: 1456), New York: Springer-Verlag, 1998, 135-146.

[11] Spires, S. V., and Goldsmith, S. Y. (1998)

Exhaustive geographic search with mobile robots along space-filling curves.

In A. Dragoul, M. Tambe, and T. Fukuda (Eds.), Collective Robotics (Lecture Notes in Artificial Intelligence: 1456), New York: Springer-Verlag, 1998, $1-12$.
[12] Burgard, W., Moors, M., and Schneider, F. (2002)

Collaborative exploration of unknown environments with teams of mobile robots.

In M. Beetz, J. Hestzberg, M. Ghallab, and M. E. Pollack (Eds.), Advances in Plan-Based Control of Robotic Agents (Lecture Notes in Computer Science: 2466), New York: Springer-Verlag, 2002.

[13] Kitamura, Y., Chauang, Z. B., Tatsumi, S., Okumoto, T., and Deen, S. M. (1993)

A cooperative search scheme for dynamic problems. In Proceedings of the IEEE Conference on Systems, Man, and Cybernetics, 1993.

[14] Dell, R. F., Eagle, J. N., Martins, G. H. A., and Santos, A. G. (1996) Using multiple searchers in constrained-path, moving-target search problems. Naval Research Logistics, 43 (1996), 463-480.

[15] Sujit, P. B., and Ghose, D. (2003)

Optimal uncertainty reduction search using the $k$-shortest path algorithm.

In Proceedings of the American Control Conference, ACC'2003, Denver, CO, June 2003, 3269-3274.

[16] Ahuja, R. K., Magnanti, T. L., and Orlin, J. B. (1993) Network Flows: Theory, Algorithms, and Applications. Englewood Cliffs, NJ: Prentice Hall, 1993.

[17] Eppstein, D. (1989) Finding the $k$-shortest paths. SIAM Journal of Computing, 28 (1989), 652-673.

[18] Jiminez, V., and Marzal, A. (1999)

Computing the $k$ shortest paths: A new algorithm and an experimental comparison.

In J. S. Vitter and C. D. Zaroliagis (Eds.), Algorithm Engineering (Lecture Notes in Computer Science: 1668), New York: Springer-Verlag, 1999, 15-29.

[19] Sharma, M. K., and Ghose, D. (2002)

Cooperative search using uninhabited autonomous air vehicles.

Technical report TR-PME-2002-09, DRDO-IISc

Programme on Advanced Research in Mathematical Engineering, Indian Institute of Science, Bangalore, India, Aug. 2002.

[20] Ghose, D., Shamma, J. S., and Speyer, J. L. (2002) Cooperative search using teams of partially connected autonomous agents: Information latency and search strategies.

Technical report, Department of Mechanical and Aerospace Engineering, University of California at Los Angeles, July 2002.

[21] Sujit, P. B., and Ghose, D. (2002)

Optimal uncertainty reduction search using the $k$-shortest path algorithm.

Technical report TR-PME-2002-18, DRDO-IISc Programme on Advanced Research in Mathematical Engineering, Indian Institute of Science, Bangalore, India, Oct. 2002. 

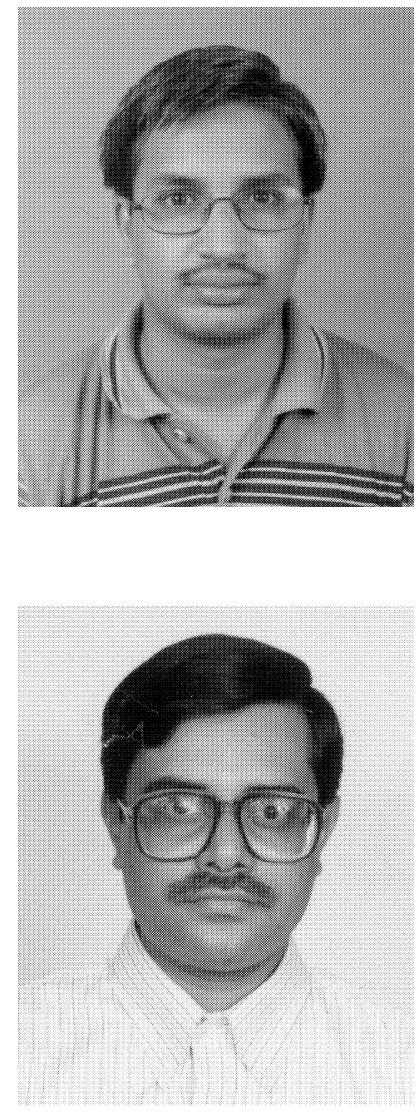

P. B. Sujit (S'03) received the B.E. degree in electrical engineering from Bangalore University, India, in 1998 and M.Tech. degree in electrical engineering from Visveswaraya Technological University, India, in 2002.

At present he is a graduate student at the Department of Aerospace Engineering, Indian Institute of Science, Bangalore. His research interests include search theory and game theory.

D. Ghose (M'03) obtained a B.Sc. (Engg.) degree from the National Institute of Technology (formerly the Regional Engineering College), Rourkela, India, in 1982, and an M.E. and a Ph.D. degree, from the Indian Institute of Science, Bangalore, in 1984 and 1990, respectively, all in electrical engineering.

$\mathrm{He}$ is presently an associate professor of Aerospace Engineering at the Indian Institute of Science. He has held visiting positions at several other universities such as the University of California at Los Angeles and the Kangwon National University in South Korea. His research interests are in guidance and control of aerospace vehicles, distributed decision-making systems, and scheduling problems in distributed computing systems.

Dr. Ghose was selected for the Alexander von Humboldt fellowship for the year 2000 . 